\title{
Regular subgroups with large intersection
}

\author{
Riccardo Aragona $^{1}$ (D) Roberto Civino ${ }^{1} \cdot$ Norberto Gavioli $^{1} \cdot$ Carlo Maria Scoppola $^{1}$
}

Received: 15 November 2018 / Accepted: 2 April 2019 / Published online: 8 April 2019

(c) Fondazione Annali di Matematica Pura ed Applicata and Springer-Verlag GmbH Germany, part of Springer Nature 2019

\begin{abstract}
In this paper, we study the relationships between the elementary abelian regular subgroups and the Sylow 2-subgroups of their normalisers in the symmetric group $\operatorname{Sym}\left(\mathbb{F}_{2}^{n}\right)$, in view of the interest that they have recently raised for their applications in symmetric cryptography.
\end{abstract}

Keywords Elementary abelian regular subgroups - Sylow 2-subgroups · Affine groups · Block ciphers · Cryptanalysis

Mathematics Subject Classification 20B35 $\cdot$ 20D20 · 94A60

\section{Introduction}

Let $n>2$ and let $(V,+)$ be an $n$-dimensional vector space over the field with two elements, where + denotes the bitwise XOR operation, i.e. the bitwise addition modulo two. The conjugacy class of elementary abelian regular subgroups of the symmetric group $\operatorname{Sym}(V)$ has recently drawn the attention of symmetric cryptographers, as these subgroups and their normalisers may be used to detect weaknesses in symmetric-encryption methods, i.e. block ciphers. More specifically, cryptanalysts may take advantage of the alternative operations that

R. Aragona, N. Gavioli, and C. M. Scoppola are members of INdAM-GNSAGA (Italy). R. Civino thankfully acknowledges support by the Department of Mathematics of the University of Trento. The authors thankfully acknowledge support by MIUR-Italy via PRIN 2015TW9LSR “Group theory and applications". Part of this work has been carried out during the cycle of seminars "Gruppi al Centro" organised at INdAM in Rome.

$凶 \quad$ Riccardo Aragona

riccardo.aragona@univaq.it

Roberto Civino

roberto.civino@univaq.it

Norberto Gavioli

norberto.gavioli@univaq.it

Carlo Maria Scoppola

scoppola@univaq.it

1 Dipartimento di Ingegneria e Scienze dell'Informazione e Matematica, Università degli Studi dell'Aquila, Via Vetoio, I-67100 L'Aquila, Coppito, Italy 
these groups induce on the plaintext space and exploit them to detect biases in the distribution of the ciphertexts.

In this paper, motivated by the possible cryptographic applications, we consider the families of maximal-intersection subgroups and second-maximal-intersection subgroups of $\operatorname{Sym}(V)$, i.e. the families of elementary abelian regular subgroups of $\operatorname{Sym}(V)$ that intersect the image $\sigma_{V}$ of the right regular representation $\sigma$, here usually denoted by $T$, in a subgroup of index two or four in $T$. We prove that each second-maximal-intersection subgroup is affine. In other words, such a subgroup is contained in $\operatorname{AGL}(V)$, the normaliser of $T$ in $\operatorname{Sym}(V)$, which is a maximal subgroup of the alternating group $\operatorname{Alt}(V)$ [16]. Moreover, we prove that every Sylow 2-subgroup of AGL $(V)$ contains one and only one second-maximal-intersection subgroup as a normal subgroup. As a consequence, we conclude that $\left[N_{\mathrm{Sym}(V)}(\Sigma): \Sigma\right]=2$, where $\Sigma$ is a Sylow 2-subgroup of $\operatorname{AGL}(V)$.

\section{Motivation and links to symmetric cryptography}

A block cipher on the plaintext space $V$ is a family $\left\{E_{k}\right\}_{k \in \mathcal{K}}$ of nonlinear permutations of $\operatorname{Sym}(V)$, called encryption functions, indexed by a set of parameters $\mathcal{K}$, called keys. Each encryption function is usually obtained as the composition of different layers, each one designed with a precise cryptographic goal, depending on its role in the employed algorithm (see, e.g. $[5,15,18])$. Some of those layers provide entropy to the encryption process by additions with round keys in $V$ computed by a public procedure, called key schedule, starting from the user-selected key in $\mathcal{K}$. The nonlinearity of the functions $E_{k}$ is one of the crucial requirements to provide security against a large variety of statistical attacks, such as differential [6] and linear [17] cryptanalysis. For this reason, ways of making the cipher's components as far as possible from being linear are extensively studied [19]. The usually considered notion of nonlinearity is given with respect to the operation which is used in the cipher to perform the key addition. However, it is worth noticing here that the notion of nonlinearity is not univocal. For example, one of the classical notions of nonlinearity for $f \in \operatorname{Sym}(V)$ is the one that measures the distance of $f$ from the set of the affine functions $\operatorname{AGL}(V)$ [7]. Another well-established definition [19] looks at the behaviour of the derivatives of $f$, measuring how far they are from being constant. Other notions of nonlinearity may be found in $[1,11]$. As already mentioned, the security of a cipher depends, among other things, on the requirement that its encryption functions do not behave as linear functions, i.e. they lie far from the set $\operatorname{AGL}(V)$. However, several isomorphic copies of $\operatorname{AGL}(V)$ are contained in $\operatorname{Sym}(V)$, and each of them corresponds to a different operation endowing $V$ with a distinct vector space structure. A target of a new branch of research in symmetric cryptography $[4,8,9,12]$ is to investigate the nonlinearity of the encryption functions of a cipher with respect to these alternative operations.

Let us recall that $\sigma: V \rightarrow \operatorname{Sym}(V)$ denotes the right regular representation and that

$$
T \stackrel{\text { def }}{=} \sigma_{V}=\left\{\sigma_{v} \mid v \in V, x \mapsto x+v\right\} .
$$

If $\tau: v \mapsto \tau_{v}$ is another embedding of $V$ in $\operatorname{Sym}(V)$ as a regular permutation subgroup, then we denote by $\tau_{V}=\left\{\tau_{v} \mid v \in V\right\}$ its image, where the map $\tau_{v}$ is the one for which $0 \mapsto v$. A new operation $\circ$ on $V$ may be defined from $\tau_{V}$ by setting

$$
\forall u, v \in V \quad u \circ v \stackrel{\text { def }}{=} u \tau_{v} .
$$

It is straightforward to check that $(V, \circ)$ is an elementary abelian 2-group. The notation $T_{\circ}$ for $\tau_{V}$, used in $[4,8,12]$, might be ambiguous in this paper. For this reason, we prefer to use 
the more explicit notation $\tau_{V}=T^{g}$, where the element $g \in \operatorname{Sym}(V)$ conjugates $T$ in $\tau_{V}$. The existence of such an element is a consequence of a result by Dixon [13], which we recall in Sect. 2.

In what follows we quickly describe the contributions of $[4,8,9,12]$ in order to give an idea of the possible attacks. Abelian regular subgroups of the affine group AGL $(V)$ are described in [9] in terms of commutative associative algebra structures defined on $V$. This is also the case for $T^{g}$. In [4,12] the authors designed a toy cipher whose set of encryption functions is contained in a conjugate $\operatorname{AGL}(V)^{g}$ for some $g \in \operatorname{Sym}(V)$. In other words, the encryption functions are affine with respect to the new operation, different from the classical bitwise XOR, defined as above from $T^{g}$. The first differential attack [6] using an alternative operation has been performed in [8], where the authors designed a cipher which is resistant to the classical differential attack with respect to + but may be attacked using another operation specifically created.

In the works $[4,8,12]$, the subspace

$$
W_{\circ} \stackrel{\text { def }}{=}\{k \in V \mid \forall x \in V \quad k \circ x=k+x\}
$$

plays an important cryptographic role, since it represents the set of round keys for which the XOR addition and the o-addition give the same result. For this reason, they are called weak keys and $W_{\circ}$ is called the weak-key subspace. The same notation is also used in this paper. It is straightforward to check that $W_{\circ}$ is a subspace of both $(V,+)$ and $(V, \circ)$ and $\sigma_{W_{\circ}}=\tau_{W_{\circ}}=T \cap T_{\circ}$.

Finally, it is worth mentioning that the cryptanalysis exploiting an operation different from the one used to perform the key addition may be a hard task, since usually the transformations which are affine with respect to the new operation may not be affine with respect to the classical one. For this reason, some modern-cipher designers decided to alternate in their algorithms several layers, each of which is affine with respect to a different operation. A classic example of this design strategy is the Russian government standard GOST [14], where an addition modulo $2^{32}$ is used besides the classical XOR for the key addition. Such a design strategy makes the use of standard cryptanalytic techniques more difficult. For this reason, in the case of GOST, only few results are known regarding the cryptanalysis, see, e.g. [2,20].

\section{Organisation of the paper}

In Sect. 2, we introduce our notation and we recall some known preliminary results. Sect. 3 is dedicated to the study of the maximal-intersection subgroups and second-maximalintersection subgroups of $\operatorname{Sym}(V)$. In our main results (Theorems 2 and 3), we parameterise such groups. In Sect. 4, we focus our attention on the case of second-maximal-intersection subgroups and on the Sylow 2-subgroups of AGL $(V)$. We show that every Sylow 2-subgroup of $\operatorname{AGL}(V)$ contains one and only one second-maximal-intersection subgroup as a normal subgroup (Theorem 4). As a consequence, we show that every Sylow 2-subgroup of AGL( $V)$ is self-normalising in $\operatorname{AGL}(V)$ and has index 2 in its normaliser in $\operatorname{Sym}(V)$ (Theorem 7). Lastly, Sect. 5 concludes the paper with some open problems.

\section{Notation and preliminary results}

We have already introduced part of our (rather standard) notation. Moreover, the set $\left\{e_{1}, e_{2}, \ldots, e_{n}\right\}$ denotes the canonical basis of $V$. For each given vector $v \in V$, we denote 
by $v^{(i)} \in \mathbb{F}_{2}$ the $i$-th coordinate of $v$, and by $v^{(i: j)} \in \mathbb{F}_{2}^{j-i+1}$ the vector composed by the coordinates of $v$ from the $i$-th to the $j$-th, for $1 \leq i<j \leq n$. If $G$ is a group acting on $V$, we denote by $v g$ the image of the action of $g \in G$ on $v \in V$. The identity element of $G$ is denoted by $1_{G}$. We also recall that the affine group $\operatorname{AGL}(V)$ is $T \rtimes \operatorname{GL}(V)$, where $\operatorname{GL}(V)$ is the group of linear bijections of $V$. The identity matrix of GL $\left(\mathbb{F}_{2}^{d}\right)$ is also denoted by $1_{d}$.

Let now $\tau$ be a regular representation as in the previous section. In this paper, we will extensively use the fact that, by [13, proof of Lemma 1], there exists $g \in \operatorname{Sym}(V)$ such that $\tau_{V}=T^{g}$. Denoting by $\tau_{v}$ the unique map of $\tau_{V}$ sending 0 to $v$, one has $\left(\sigma_{v}\right)^{g}=\tau_{\left(0 g^{-1}+v\right) g}$ for all $v \in V$. For the convenience of the reader, the proof of Dixon's result is reproduced here.

Theorem 1 Let $X=\left\{x_{1}, x_{2}, \ldots, x_{m}\right\}$ be a finite set and let $H$ and $K$ be regular subgroups of $\operatorname{Sym}(X)$. If $H \cong K$, then there exists $g \in \operatorname{Sym}(X)$ such that $K=H^{g}$.

Proof Let $\zeta: H \rightarrow K$ be an isomorphism. Since both the groups are regular, we have that $\left\{x_{1} h \mid h \in H\right\}=\left\{x_{1} k \mid k \in K\right\}=X$. Let us now define the permutation $g \in \operatorname{Sym}(X)$ by setting $\left(x_{1} h\right) g \stackrel{\text { def }}{=} x_{1}(h \zeta)$, for each $h \in H$. The result follows from the fact that $h^{g}=h \zeta$ for all $h \in H$. Indeed, let $1 \leq i \leq m$ and let $h^{\prime} \zeta \in K$ such that $x_{1} h^{\prime} \zeta=x_{i}$, where $h^{\prime} \in H$. Then, since $\zeta$ is an isomorphism, we have

$$
x_{i} h^{g}=x_{i} g^{-1} h g=\left(x_{1} h^{\prime} \zeta\right) g^{-1} h g=\left(x_{1} h^{\prime} h\right) g=x_{1}\left(h^{\prime} h \zeta\right)=x_{1} h^{\prime} \zeta h \zeta=x_{i} h \zeta .
$$

Since the property holds for each $1 \leq i \leq m$, then for each $h \in H$, we have $h^{g}=h \zeta$, therefore $H^{g}=H \zeta=K$.

The following lemma generalises a well know fact.

Lemma 1 Every elementary abelian regular subgroup of $\operatorname{Sym}(V)$ is the unique non-trivial proper normal subgroup of its own normaliser.

Proof By [13], the result may be proven for the regular group $T$, up to conjugation. Since $T$ is well known to be a self-centralising minimal normal subgroup of $\operatorname{AGL}(V)$, if $\bar{T} \neq T$ is a minimal normal subgroup of $\operatorname{AGL}(V)$, then $T \cap \bar{T}=\left\{1_{\operatorname{Sym}(V)}\right\}$. So that $\bar{T}$ centralises $T$, from which it follows that $\bar{T}<T$, a contradiction. Now, let $N$ be a non-trivial normal subgroup of $\operatorname{AGL}(V)$. By the previous argument, $T \leq N$ and $N / T \unlhd \operatorname{AGL}(V) / T \cong \operatorname{GL}(V)$, which is simple since $\operatorname{dim}(V)>2$ and the ground field has characteristic 2. Therefore, $N=\operatorname{AGL}(V)$.

The following remark will be useful to describe the centraliser over $\operatorname{Sym}(V)$ of a subgroup of $T$.

Remark 1 Let $H$ be a group acting transitively on a set $X$ and let $Z \stackrel{\text { def }}{=} X \times Y$, where $Y$ is a set. There exists a canonical embedding $\theta: H \rightarrow \operatorname{Sym}(Z)$ under which the action of $H$ is defined by $(x, y) h^{\theta}=(x h, y)$. Besides, there exists another embedding

$$
\operatorname{Sym}(X)^{Y} \hookrightarrow \operatorname{Sym}(Z)
$$

defined by $(x, y) f=\left(x f_{y}, y\right)$, where $f \in \operatorname{Sym}(X)^{Y}$ is the function sending $y \mapsto f_{y} \in$ $\operatorname{Sym}(X)$. In particular, $C_{\operatorname{Sym}(X)}(H)^{Y}$ is a subgroup of $\operatorname{Sym}(Z)$ which centralises $H^{\theta}$. Notice that also $\operatorname{Sym}(Y)$ embeds in $\operatorname{Sym}(Z)$ by way of $(x, y) \pi=(x, y \pi)$, for $\pi \in \operatorname{Sym}(Y)$. This group centralises $H^{\theta}$ and normalises and intersects trivially $C_{\operatorname{Sym}(X)}(H)^{Y}$. As a consequence we have

$$
C_{\operatorname{Sym}(X)}(H)^{Y} \rtimes \operatorname{Sym}(Y)=C_{\operatorname{Sym}(X)}(H){ }_{2} \operatorname{Sym}(Y) \leq C_{\operatorname{Sym}(Z)}\left(H^{\theta}\right) .
$$


It is straightforward, but somewhat lengthy, to show that the opposite inclusion holds, i.e.

$$
C_{\operatorname{Sym}(Z)}\left(H^{\theta}\right)=C_{\operatorname{Sym}(X)}(H) \imath_{Y} \operatorname{Sym}(Y) .
$$

As a consequence of the previous remark, we can prove the following.

Lemma 2 Let $G$ be a finite group and $\sigma, \lambda: G \rightarrow \operatorname{Sym}(G)$, respectively, be the right and left regular representations of $G$. If $H \unlhd G$, then

$$
C_{\text {Sym }(G)}\left(H^{\sigma}\right)=H^{\lambda} 2 \operatorname{Sym}(G / H),
$$

where $G / H$ is the set of left cosets of $H$ in $G$.

Proof It is well known that $C_{\mathrm{Sym}(H)}\left(H^{\sigma}\right)=H^{\lambda}$; therefore, the claim follows from Eq. (1). $\square$

Corollory 1 If $M$ is a subgroup of $T$ of order $2^{n-m}$, then $C_{\operatorname{Sym}(V)}(M)$ is the wreath product $M 2 \operatorname{Sym}\left(2^{m}\right)$. In particular $\left|C_{\operatorname{Sym}(V)}(M)\right|=2^{m} ! 2^{2^{m}(n-m)}$.

Proof The result follows from Lemma 2.

Indeed, let $M_{0} \leq V$ be such that $\left(M_{0}\right)^{\sigma}=M$; then, since $V$ is abelian, $M=\left(M_{0}\right)^{\sigma}=$ $\left(M_{0}\right)^{\lambda}$.

\section{Elementary abelian regular subgroups whose intersection with the translation group is large}

In this section, we prove some results on elementary abelian regular subgroups of $\operatorname{Sym}(V)$, and more generally on fixed-point-free involutions. Our interest is, in particular, in the connections between such groups and $\operatorname{AGL}(V)$. We now parameterise the elementary abelian regular subgroups of $\operatorname{Sym}(V)$ according to the size of their intersection with $T$.

\subsection{Maximal intersection}

Here we prove that none of the aforementioned groups has a maximal intersection with $T$. The result is a consequence of the following proposition, which slightly generalises a result appearing in [12].

Proposition 1 Let $g \in \operatorname{Sym}(V)$ such that $T \neq T^{g}$. If $W \leq V$ such that $\sigma_{W}=T \cap T^{g}$, then $\operatorname{dim}(W) \leq n-2$.

Proof Assume by way of contradiction that $\operatorname{dim}(W)=n-1$. Let $\left\{v_{i}\right\}_{i=1}^{n-1}$ be a basis for $W$ and $v \in V \backslash W$. The claim holds if $a \tau_{v}=a \sigma_{v}$ for any $a \in V$. If $a \in W$, there is nothing to prove; hence, without loss of generality, we may assume $a=w+v$, for some $w \in W$. Then,

$$
\begin{aligned}
a \tau_{v} & =(w+v) \tau_{v}=\left(w \sigma_{v}\right) \tau_{v} \\
& =\left(w \tau_{v}\right) \tau_{v}=w\left(\tau_{v}\right)^{2}=w \\
& =a \sigma_{v} .
\end{aligned}
$$

From Proposition 1, we can derive the following result.

Theorem 2 Let $M$ be any maximal subgroup of T. Then, $C_{\operatorname{Sym}(V)}(M)<\operatorname{AGL}(V)$. Moreover, $\left|C_{\text {Sym }(V)}(M)\right|=2^{2 n-1}$. 
Proof It is enough to show that $T \unlhd C_{\mathrm{Sym}(V)}(M)$, so that $C_{\mathrm{Sym}(V)}(M)$ is a subgroup of the normaliser of $T$ which is $\operatorname{AGL}(V)$. To this purpose, let $c \in C_{\operatorname{Sym}(V)}(M)$ and let $\xi$ be any translation in $T \backslash M$. The aim is to show that $\eta \stackrel{\text { def }}{=} c^{-1} \xi c \in T$. Assume the contrary by way of contradiction, so that $\eta \notin T$. Clearly, $\langle M, \eta\rangle=\langle M, \xi\rangle^{c}=T^{c}$ and $|(T \cap\langle M, \eta\rangle)|=$ $2^{n-1}$. This contradicts the previous proposition. The claim $\left|C_{\operatorname{Sym}(V)}(M)\right|=2^{2 n-1}$ follows from Corollary 1.

\subsection{Second-maximal intersection}

In this section, we prove that elementary abelian regular subgroups that intersect $T$ in a second-maximal subgroup are all contained in $\operatorname{AGL}(V)$.

From now on, we shall assume that $g \in \operatorname{Sym}(V)$ is such that $\sigma_{W}=T \cap T^{g}$, and $\operatorname{dim}(W)=n-2$, where $W \leq V$. Let $W_{1} \stackrel{\text { def }}{=} W+v_{1}, W_{2} \stackrel{\text { def }}{=} W+v_{2}$ and $W_{1,2} \stackrel{\text { def }}{=} W+v_{1}+v_{2}$, for some $v_{1}$ and $v_{2}$ in $V$ which are linearly independent modulo $W$, be the non-trivial cosets of $W$ in $V$. Notice that any element in $\operatorname{Sym}(V)$ centralising $T \cap T^{g}$ permutes the cosets of $W$ in $V$.

In the hypothesis of this section, Theorem 2 has not a counterpart. However, we have the following generalisation.

Lemma 3 Let $W \leq V$ be such that $\operatorname{dim}(W)=n-2$. Let $\varphi \in \operatorname{Sym}(V)$ be an involution centralising the second-maximal subgroup $\sigma_{W}$ of $T$ and also not fixing any of the cosets of $W$. Then, $\varphi \in \operatorname{AGL}(V)$.

Proof Since $\varphi$ is regular and it centralises $\sigma_{W}$, its action on $V$ is completely determined by its action on the cosets of $W$, which we may assume being as the involution $\left(W, W_{1}\right)\left(W_{2}, W_{1,2}\right)$, and by the choices of $0 \varphi$ and $v_{2} \varphi$. Indeed, if $0 \neq u \in W$, then $u \varphi$ is determined as $u \varphi=$ $0 \sigma_{u} \varphi=0 \varphi \sigma_{u} \in W_{1}$. Similarly, $v_{2} \varphi$ determines the action of $\varphi$ on each element of $W_{2}$. Set now $z \stackrel{\text { def }}{=} 0 \varphi \in W_{1}, x \stackrel{\text { def }}{=} v_{1} \varphi+z+v_{1}$, and $y \stackrel{\text { def }}{=} v_{2} \varphi+z+v_{2}$. Let $\bar{\varphi}$ be the affinity sending $w+\alpha v_{1}+\beta v_{2} \mapsto w+\alpha v_{1}+\beta v_{2}+\alpha x+\beta y+z$, where $\alpha, \beta \in \mathbb{F}_{2}$. Then, an easy check shows that $0 \bar{\varphi}=z, v_{2} \bar{\varphi}=v_{2} \varphi$, and that $\bar{\varphi}$ is an involution which centralises $\sigma_{W}$ and acts on the cosets of $W$ as the involution $\left(W, W_{1}\right)\left(W_{2}, W_{1,2}\right)$. Therefore, $\bar{\varphi}=\varphi$, which is what we meant to prove.

In the very special case $n=3$, every fixed-point-free involution in $\operatorname{Sym}(V)$ centralising $\sigma_{W}$ is affine. Indeed, since $n=3, W=\langle w\rangle$. Now, if $\varphi$ fixes all the four cosets of $W$, then $\varphi$ is the translation $\sigma_{w}$. If $\varphi$ acts on the cosets without fixed points, then Lemma 3 applies. Finally, we are left with the case where two cosets are fixed and two are exchanged. Assuming without loss of generality that $\varphi$ acts on the cosets as $\left(W, W_{1}\right)$, then $\varphi$ is the affinity sending $\left(\gamma w+\alpha v_{1}+\beta v_{2}\right) \mapsto(\gamma+\alpha) w+(\alpha+\beta+1) v_{1}+\beta v_{2}$, where $\alpha, \beta, \gamma \in \mathbb{F}_{2}$. A straightforward check may be also performed using MAGMA [3].

Remark 2 In the following theorem, we will use a well-known fact: if $G<\operatorname{Sym}(V)$ is a regular subgroup and $H \unlhd G$, then $G / H$ acts regularly on the set of the orbits of $H$.

The following straightforward consequence of Lemma 3 and Remark 2 is the main contribution of this section. We, however, include a second constructive proof, in view of its use in the remainder of the paper. The notation used here and below is the one specified at the beginning of Sect. 2 .

Theorem 3 Let $g \in \operatorname{Sym}(V)$ and $W \leq V$ such that $\sigma_{W}=T \cap T^{g}$. If $\operatorname{dim}(W)=n-2$, then $T^{g}<\operatorname{AGL}(V)$. 
Proof It is enough to show that if $T^{g}=\left\langle\pi, \varepsilon, \sigma_{W}\right\rangle$, then $\pi, \varepsilon \in \operatorname{AGL}(V)$. This is granted by Lemma 3 , since $\pi$ and $\varepsilon$ are regular involutions centralising $\sigma_{W}$ and not fixing any of its cosets.

Alternatively, we now construct explicitly two affinities $\bar{\pi}$ and $\bar{\varepsilon}$ which are, respectively, congruent to $\pi$ and $\varepsilon$ modulo translations in $\sigma_{W}$. A similar construction is provided in [12]. Let us assume $W$ is spanned by the last $n-2$ vectors of the canonical basis of $V$. Notice that $\pi, \varepsilon \in T^{g}$, thus

(1) $\pi$ and $\varepsilon$ are fixed-point-free,

(2) $\pi$ and $\varepsilon$ are involutions,

(3) $\pi, \varepsilon \in C_{\operatorname{Sym}(V)}\left(\sigma_{W}\right)$,

(4) $\pi \varepsilon=\varepsilon \pi$.

Moreover, since $T^{g}$ is regular, by Remark 2, it is then possible to assume that $\pi$ acts on the cosets of $W$ as the involution $\left(W, W_{1}\right)\left(W_{2}, W_{1,2}\right)$, whereas $\varepsilon$ acts as $\left(W, W_{2}\right)\left(W_{1}, W_{1,2}\right)$. Up to a composition by a translation in $\sigma_{W}$, one can assume that $v_{2} \pi=v_{1}+v_{2}$, so that the action of $\pi$ on $V$ is completely determined by the value of $0 \pi \in W_{1}$. Similarly, modulo a translation in $\sigma_{W}$, let us assume that $v_{1} \varepsilon=v_{1}+v_{2}$. Consequently, since $T^{g}=\left\langle\pi, \varepsilon, \sigma_{W}\right\rangle$, the action of $T^{g}$ on $V$ is completely determined by $W$ and by the values of $0 \pi, 0 \varepsilon$, and $0 \pi \varepsilon$. What remains to be proven is that for each possibile choice of $0 \pi$ in $W_{1}, 0 \varepsilon$ in $W_{2}$ and $0 \pi \varepsilon$ in $W_{1,2}$ there exist two affinities $\bar{\pi}, \bar{\varepsilon} \in \operatorname{AGL}(V)$ such that for each $v \in V$, we have $v \pi=v \bar{\pi}$ and $v \varepsilon=v \bar{\varepsilon}$. Consider now the functions $\bar{\pi}$ sending $x \mapsto x+x^{(2)} b+0 \pi$ and $\bar{\varepsilon}$ sending $x \mapsto x+x^{(1)} b+0 \varepsilon$, where $b=0 \pi+0 \varepsilon+0 \pi \varepsilon$ and $x^{(i)}$ denotes the $i$-th coordinate of $x$. Then, $\bar{\pi}, \bar{\varepsilon} \in \operatorname{AGL}(V)$ satisfy the four properties listed above and $0 \bar{\pi}=0 \pi, v_{2} \bar{\pi}=v_{1}+v_{2}$ $\bmod W, 0 \bar{\varepsilon}=0 \varepsilon, v_{1} \bar{\varepsilon}=v_{1}+v_{2} \bmod W$. Therefore, $\pi=\bar{\pi}$ and $\varepsilon=\bar{\varepsilon}$; hence, the desired result is proved.

Remark 3 In the hypotheses of Theorem 3, by interchanging the roles of $T$ and $T^{g}$, one can easily obtain that also $T$ is a subgroup of $\operatorname{AGL}(V)^{g}$, i.e. $T$ normalises $T^{g}$.

It is convenient to give a more practical representation of the groups $T^{g}$ such that $\operatorname{dim}(W)=n-2$, where $\sigma_{W}=T \cap T^{g}$. From now on, we assume that $W$ is spanned by the last $n-2$ vectors of the canonical basis of $V$. The next result gives a parameterisation and counts the number of subgroups with such a property.

Proposition 2 Let $W \stackrel{\text { def }}{=}\left\langle e_{i} \mid 3 \leq i \leq n\right\rangle$. For $b \in W \backslash\{0\}$, the subgroups

$$
T_{b} \stackrel{\text { def }}{=}\left\langle\pi_{b}, \varepsilon_{b}, \sigma_{e_{i}} \mid 3 \leq i \leq n\right\rangle,
$$

where

$$
\pi_{b}=\left(\begin{array}{c|c}
1_{2} & 0 \\
\hline 0 & b^{(3: n)}
\end{array}\right) \sigma_{e_{1}}, \quad \varepsilon_{b}=\left(\begin{array}{c|c}
1_{2} & b^{(3: n)} \\
\hline 0 & 0 \\
\hline 0 & 1_{n-2}
\end{array}\right) \sigma_{e_{2}},
$$

are exactly the elementary abelian regular subgroups $\bar{T}$ of $\operatorname{Sym}(V)$ such that $T \cap \bar{T}=\sigma_{W}$. In particular, there are $2^{n-2}-1$ such subgroups.

Proof As in the proof of Theorem 3, we necessarily have $\bar{T}=T_{b}$, for some $b \in W \backslash\{0\}$. Note that $b$ is completely determined by the fact that $x \pi+x+0 \pi$ is a scalar multiple of $b$ for any $\pi \in T_{b}$. Hence, the desired result follows from the fact that such subgroups are in one-to-one correspondence with the possible choices of $b \in W \backslash\{0\}$. 
The general problem of parameterising all the elementary abelian regular subgroups $\bar{T}$ of $\operatorname{Sym}(V)$ and of $\operatorname{AGL}(V)$ according to the size of their intersection with $T$ is not easy in general. Proposition 2 solves this problem in the case of second-maximal-intersection subgroups. Partial results have been obtained in the case $\bar{T}<\operatorname{AGL}(V)$ [4,8,12]. In [12], a result similar to the following corollary is proved, where $\operatorname{AGL}(V)$ appears in place of $\operatorname{Sym}(V)$. The present form is a consequence of Theorem 3, and the result is easily derived by Proposition 2.

Corollory 2 The group $\operatorname{Sym}(V)$ contains $t_{n}$ elementary abelian regular subgroups whose intersection with $T$ is a second-maximal subgroup of $T$, where

$$
t_{n} \stackrel{\text { def }}{=} \frac{\left(2^{n-2}-1\right)\left(2^{n-1}-1\right)\left(2^{n}-1\right)}{3} .
$$

Proof The integer $t_{n}$ may be obtained as the product of $2^{n-2}-1$ and $\left(2^{n}-1\right)\left(2^{n}-2\right) / 6$, respectively, the number of elementary abelian regular subgroups which intersect $T$ in the subspace spanned by the last $n-2$ vectors of the canonical basis and the number of $(n-2)$ dimensional subspaces of $V$.

\section{Sylow 2-subgroups of $A G L(V)$}

In this section, $S$ will denote a Sylow 2-subgroup of $\operatorname{AGL}(V)$. Up to a conjugation with an element in $\operatorname{AGL}(V)$, we can assume

$$
S \stackrel{\text { def }}{=}\left\{U \sigma_{v} \mid U \in \mathcal{U}, v \in V\right\}
$$

where $\mathcal{U}$ is the group of upper unitriangular matrices, a Sylow 2-subgroup of GL( $V)$. Notice that $\mathcal{U}$ is generated by the matrices $1_{n}+E_{i, i+1}$, where $1 \leq i \leq n-1$ and $E_{i, j}$ is the matrix whose entries are all zero except the $(i, j)$-th entry which is 1 .

Remark 4 The action by conjugation of $S$ on $T$ is canonically identified as the action of $\mathcal{U}$ on $V$. As a $\mathcal{U}$-module, $V$ is uniserial, i.e. any $\mathcal{U}$-submodule of $V$ belongs to the maximal flag $\{0\}=V_{0}<V_{1}<\ldots<V_{n}=V$, where $V_{i}=\left\langle e_{n-i+1}, \ldots, e_{n}\right\rangle$ and $1 \leq i \leq n$. Conversely, for each given maximal flag $\mathcal{F}$ whose members are

$$
\{0\}=V_{0}<V_{1}<\ldots<V_{n}=V,
$$

if $\mathcal{U}$ is the stabiliser of $\mathcal{F}$ in $\operatorname{GL}(V)$, then $\mathcal{U} T$ is a Sylow 2-subgroup of $\operatorname{AGL}(V)$. The previous construction yields a one-to-one correspondence between Sylow 2-subgroups of $\operatorname{AGL}(V)$ and the set of maximal flags of subspaces of $V$. Indeed, given a maximal flag $V_{0}<V_{1}<\ldots<V_{n}$, the corresponding Sylow 2-subgroup is exactly the stabiliser by conjugation of $\left\{1_{\operatorname{Sym}(V)}\right\}=\sigma_{V_{0}}<\sigma_{V_{1}}<\ldots<\sigma_{V_{n}}=T$. This fact is used throughout this section without any further reference.

The following theorem is crucial in this section.

Theorem 4 Every Sylow 2-subgroup $\Sigma$ of $\operatorname{AGL}(V)$ contains exactly one elementary abelian regular subgroup $T_{\Sigma}$ intersecting $T$ in a second-maximal subgroup of $T$ and which is normal in $\Sigma$.

Proof Since $T \unlhd$ AGL $(V)$, then $T$ is a normal subgroup of every Sylow 2-subgroup of $\operatorname{AGL}(V)$. Without loss of generality we may assume $\Sigma$ to be the Sylow 2-subgroup $S$ defined 
in Eq. (3) and $W=\left\langle e_{i} \mid 3 \leq i \leq n\right\rangle$. Indeed, $W$ has to be the only subgroup of $V$ of order $2^{n-2}$ such that $\sigma_{W}=T \cap T_{S}$ is normalised by $S$. We denote by $\Theta$ the family of groups $\left\{T_{b} \mid b \in W \backslash\{0\}\right\}$, where

$$
T_{b}=\left\langle\pi_{b}, \varepsilon_{b}, \sigma_{W}\right\rangle<S
$$

and $\pi_{b}$ and $\varepsilon_{b}$ are as in the statement of Proposition 2. Since $|\Theta|$ is odd and $S$ fixes $T$ by conjugation, then there exists $b \in W \backslash\{0\}$ such that $S$ fixes $T_{b}$ by conjugation, i.e. $T_{b} \unlhd S$. It remains to be proven that such a group is unique. Now, we prove that the unique $b \in W$ that corresponds to a normal subgroup in $S$ is $(0,0, \ldots, 0,1)$, from which the desired result follows. First, by Remark 3, we have $T<N_{\operatorname{Sym}(V)}\left(T_{b}\right)$ for every $b \in W$, so each $\sigma_{v} \in T<S$ normalises $T_{b}$. Moreover, any upper unitriangular matrix in $\mathcal{U}$ fixes by conjugation $\sigma_{W}$, indeed if $U \in \mathcal{U}$, then $\sigma_{e_{i}}^{U}=\sigma_{e_{i} U}$ and $e_{i} U \in W$ for every $3 \leq i \leq n$. Therefore, we are left to determine $b$ such that $v^{U} \in T_{b}$, for every $v \in\left\{\pi_{b}, \varepsilon_{b}, \pi_{b} \varepsilon_{b}\right\}$ and $U \in \mathcal{U}$. Note that $\mu \in T_{b}$ implies that $x+x \mu+0 \mu=\alpha b$, for all $x \in V$ and for some scalar $\alpha \in \mathbb{F}_{2}$. Now, for $\mu=\pi_{b}$,

$$
\begin{aligned}
x \mu^{U}=x U^{-1} \pi_{b} U & =\left(x U^{-1}+\left(x U^{-1}\right)^{(2)} b+e_{1}\right) U \\
& =x U^{-1} U+\left(x U^{-1}\right)^{(2)} b U+e_{1} U \\
& =x+\left(x U^{-1}\right)^{(2)} b U+e_{1} U .
\end{aligned}
$$

Hence, $\pi_{b}^{U} \in T_{b}$ implies $\alpha b=x+x \pi_{b}^{U}+0 \pi_{b}^{U}=\left(x U^{-1}\right)^{(2)} b U$. As a consequence, $b$ is a common eigenvector for the elements of $\mathcal{U}$; therefore, $b=(0,0, \ldots, 0,1)$.

The previous theorem has the following converse. The same notation is used.

Proposition 3 If $\bar{T}$ is an elementary abelian regular subgroup of $\operatorname{AGL}(V)$ such that $|\bar{T} \cap T|=2^{n-2}$, then there exists a Sylow 2-subgroup $\Sigma$ of AGL $(V)$ such that $\bar{T}=T_{\Sigma} \unlhd \Sigma$.

Proof Up to conjugation in GL $(V)$ we may assume that $T \cap \bar{T}$ is the image of $\left\langle e_{i} \mid 3 \leq i \leq n\right\rangle$ under $\sigma$. By Proposition 2, there exists $b \in W \backslash\{0\}$ such that $\bar{T}=T_{b}$, where $W<$ $V$ is the subspace defined by $\sigma_{W}=\bar{T} \cap T$. We need to prove that there exists a Sylow 2 -subgroup $\Sigma<\operatorname{AGL}(V)$ such that $T_{b}=T_{\Sigma}$. There exists a basis of $W$ of the form $\left\{\bar{e}_{3}, \ldots, \bar{e}_{n-1}, \bar{e}_{n}=b\right\}$ which can be extended to a basis $\left\{{\overline{e_{1}}}_{\overline{e_{2}}}, \ldots, \bar{e}_{n-1}, b\right\}$ of $V$. Let $\bar{V}_{0}=\{0\}$ and $\bar{V}_{i}=\left\langle\bar{e}_{n-i+1}, \ldots, \bar{e}_{n}\right\rangle$, where $1 \leq i \leq n$, and let $\Sigma \stackrel{\text { def }}{=} T \rtimes \overline{\mathcal{U}}$, where $\overline{\mathcal{U}}$ is the stabiliser of the flag $\left\{\bar{V}_{i}\right\}_{i=0}^{n}$. Let $L \in \mathrm{GL}(V)$ be such that $\bar{e}_{i}=e_{i} L$ for $1 \leq i \leq n$. Clearly, $\overline{\mathcal{U}}=\mathcal{U}^{L}, \Sigma=S^{L}$, and therefore $T_{b}=T_{S}{ }^{L}$, by the definition of $T_{b}$. Since $T_{S} \unlhd S$, then $T_{b} \unlhd \Sigma$. Hence, $\bar{T}=T_{b}=T_{\Sigma}$ by Theorem 4 .

We prove in Theorem 6 that if a Sylow 2-subgroup $\Sigma$ of $\operatorname{AGL}(V)$ contains a conjugate in $\operatorname{Sym}(V)$ of $T$ as a normal subgroup, then such a subgroup is either $T$ or $T_{\Sigma}$, where $T_{\Sigma}$ is as in Theorem 4. In order to do that, the following result is helpful.

Theorem 5 Let $\Sigma$ be a Sylow 2-subgroup of $\operatorname{AGL}(V)$ and $\{0\}=V_{0}<V_{1}<\ldots<V_{n}=V$ be the associated invariant flag as in Remark 4. There exist $2^{d\left(\begin{array}{c}n-d \\ 2\end{array}\right)}$ subgroups $T^{g} \leq \Sigma$, where $g \in \operatorname{Sym}(V)$, such that $\sigma_{V_{d}} \leq T \cap T^{g}$ and $T \leq N_{\operatorname{Sym}(V)}\left(T^{g}\right)=\operatorname{AGL}(V)^{g}$.

Proof We shall use the canonical embedding of $\operatorname{AGL}(V)$ in $\operatorname{GL}\left(\mathbb{F}_{2}^{n+1}\right)$ sending the affinity $\varphi: x \mapsto x L+v$ into the linear map represented by the matrix

$$
\left(\begin{array}{l|l}
1 & v \\
\hline 0 & L
\end{array}\right) .
$$


The action of the affinity $\varphi$ can be recovered by the equality

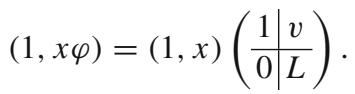

Under this monomorphism the elements of $T$ are represented by the matrices in which $L=1_{n}$. Assume now that $W=V_{d}$ is defined as in Remark 4 and that, with the same notation, $U=U_{d} \stackrel{\text { def }}{=}\left\langle v_{1}, \ldots, v_{n-d}\right\rangle$ so that $V=U \oplus W$. Taking into account this decomposition, the matrices representing elements of $\operatorname{AGL}(V)$ can be written in the block form

$$
M \stackrel{\text { def }}{=}\left(\begin{array}{l|l|l}
1 & u & w \\
\hline 0 & A & B \\
\hline 0 & D & C
\end{array}\right)
$$

where $u \in U$ and $w \in W$ (each of them referred to the relevant bases). Under this notation the elements of $\Sigma$ are represented by matrices of the form

$$
X \stackrel{\text { def }}{=}\left(\begin{array}{l|l|l}
1 & u & w \\
\hline 0 & A & B \\
\hline 0 & 0 & C
\end{array}\right)
$$

where $A$ and $C$ are upper unitriangular matrices, i.e. $X$ is an upper unitriangular matrix. Finally the matrices representing the elements $\sigma_{z}$ in $\sigma_{W}$, where $z \in W$, are those of the form

$$
Y_{z} \stackrel{\text { def }}{=}\left(\begin{array}{c|c|c}
1 & 0 & z \\
\hline 0 & 1_{n-d} & 0 \\
\hline 0 & 0 & 1_{d}
\end{array}\right) .
$$

Now we look for matrices $X$ commuting with $Y_{z}$ independently of the choice of $z$. The condition $X Y_{z}=Y_{z} X$ gives $z C=z$ for all $z \in W$, so that $C=1_{d}$.

We have shown that

$$
C_{\Sigma}\left(\sigma_{W}\right)=\left\{\left(\begin{array}{c|c|c}
1 & u & w \\
\hline 0 & A & B \\
\hline 0 & 0 & 1_{d}
\end{array}\right) \mid A \text { upper unitriangular, } u \in U \text { and } w \in W\right\} .
$$

Next, we determine the transitive elementary abelian subgroups of $C_{\Sigma}\left(\sigma_{W}\right)$ containing $\sigma_{W}$. Let $\bar{T}$ be one of them; it is well known that $\bar{T}$ has to be a regular permutation group. Let

$$
\bar{X}=\left(\begin{array}{c|c|c}
1 & u & w \\
\hline 0 & A & B \\
\hline 0 & 0 & 1_{d}
\end{array}\right) \in \bar{T} \backslash \sigma_{W}
$$

Note that $\bar{X}$ represents an affinity sending 0 to $(u, w)$. Up to the right multiplication by $Y_{w}$ we can restrict our attention to the case when $w=0$. In this case, the regularity implies that

$$
\bar{X}_{(u, 0)} \stackrel{\text { def }}{=}\left(\begin{array}{c|c|c}
1 & u & 0 \\
\hline 0 & A_{u} & B_{u} \\
\hline 0 & 0 & 1_{d}
\end{array}\right)
$$

is uniquely determined by the image $u$ of 0 under the affinity represented by $\bar{X}_{(u, 0)}$. This gives rise to a bijection from $U \oplus W$ onto $\bar{T}$ defined by

$$
(u, w) \mapsto \bar{X}_{(u, w)} \stackrel{\text { def }}{=}\left(\begin{array}{c|c|c}
1 & u & w \\
\hline 0 & A_{u} & B_{u} \\
\hline 0 & 0 & 1_{d}
\end{array}\right)
$$


where $A_{u A_{u^{\prime}}+u^{\prime}}=A_{u} A_{u^{\prime}}$ and $B_{u A_{u^{\prime}}+u^{\prime}}=B_{u}+A_{u} B_{u^{\prime}}$ for all $u, u^{\prime} \in U$. Since $\sigma_{W}$ is central in $\bar{T}$, the requirement that $\bar{T}$ is abelian is equivalent to the condition $\bar{X}_{(u, 0)} \bar{X}_{\left(u^{\prime}, 0\right)}=$ $\bar{X}_{\left(u^{\prime}, 0\right)} \bar{X}_{(u, 0)}$ for all $u, u^{\prime} \in U$. This in turn is equivalent to the following set of conditions

$$
\begin{aligned}
u^{\prime}+u A_{u^{\prime}} & =u+u^{\prime} A_{u} \\
u B_{u^{\prime}} & =u^{\prime} B_{u} \\
A_{u} A_{u^{\prime}} & =A_{u^{\prime}} A_{u} \\
A_{u} B_{u^{\prime}}+B_{u} & =A_{u^{\prime}} B_{u}+B_{u^{\prime}}
\end{aligned}
$$

for all $u, u^{\prime} \in U$. If we furthermore assume that $\bar{T}$ is normalised by $T$ we have

$$
\begin{gathered}
\bar{T} \ni\left(\begin{array}{c|c|c}
1 & u^{\prime} & 0 \\
\hline 0 & 1_{n-d} & 0 \\
\hline 0 & 0 & 1_{d}
\end{array}\right)^{-1} \bar{X}_{(u, 0)}\left(\begin{array}{c|c|c}
1 & u^{\prime} & 0 \\
\hline 0 & 1_{n-d} & 0 \\
\hline 0 & 0 & 1_{d}
\end{array}\right) \\
=\left(\begin{array}{c|c|c|c|c}
1 & u+u^{\prime}+u^{\prime} A_{u} & u^{\prime} B_{u} \\
\hline 0 & A_{u} & B_{u} \\
\hline 0 & 0 & 1_{d}
\end{array}\right)=\bar{X}_{(u, 0)}\left(\begin{array}{c|c|c|c}
1 & u^{\prime}+u^{\prime} A_{u} & u^{\prime} B_{u} \\
\hline 0 & 1_{n-d} & 0 \\
\hline 0 & 0 & 1_{d}
\end{array}\right) .
\end{gathered}
$$

It follows that

$$
\left(\begin{array}{c|c|c}
1 & u^{\prime}+u^{\prime} A_{u} & u^{\prime} B_{u} \\
\hline 0 & 1_{n-d} & 0 \\
\hline 0 & 0 & 1_{d}
\end{array}\right) \in T \cap \bar{T}=\sigma_{W},
$$

so that $u^{\prime}+u^{\prime} A_{u}=0$ for all $u \in U$ and $u^{\prime} \in U$, i.e. $A_{u}=1_{n-d}$ for all $u \in U$. As a consequence Eqs. (5), (6), (7), and (8) reduce to Eq. (6), and the map $u \mapsto B_{u}$ is linear. This incidentally proves that the map defined in Eq. (4) is an isomorphism, when $\bar{T}$ is normalised by $T$. Finally, $\bar{T}$ is elementary abelian if and only if $\bar{X}_{(u, 0)}^{2}=1_{n+1}$ for all $u \in U$. This final condition can be stated equivalently in the form

$$
u B_{u}=0 \text { for all } u \in U .
$$

The group $\bar{T}$ is then uniquely determined by the linear map $u \mapsto B_{u}$, which in turn is defined once for all $1 \leq i \leq n-d$ the matrix $B_{e_{i}}$ is given. Such matrices have to satisfy Eq. (6) and of Eq. (9), i.e. the $i$-th row of $B_{e_{j}}$ is equal to the $j$-th row of $B_{e_{i}}$ and the $i$-th row of $B_{e_{i}}$ is the zero row for $1 \leq i, j \leq n-d$. Thus, the total number of possible choices for $\bar{T}$ is easily seen to be $2^{d\left(\begin{array}{c}n-d \\ 2\end{array}\right)}$.

Theorem 6 Let $g \in \operatorname{Sym}(V)$ and let $\Sigma$ be a Sylow 2-subgroup of AGL $(V)$ containing $T^{g}$. The subgroup $T^{g}$ is normal in $\Sigma$ if and only if $T^{g} \in\left\{T, T_{\Sigma}\right\}$.

Proof We shall use the same notation as in the proof of the previous theorem. If $T^{g}$ is normal in $\Sigma$ then $T \cap T^{g}=\sigma_{V_{d}}$ for some $1 \leq d \leq n, d \neq n-1$. Notice that $T^{g}<\Sigma$, so $T$ normalises $T^{g}$. Thus,

$$
T^{g}=\bar{T}=\left\{\left(\begin{array}{c|c|c}
1 & u & w \\
\hline 0 & 1_{n-d} & B_{u} \\
\hline 0 & 0 & 1_{d}
\end{array}\right) \mid u \in U\right\}
$$

is determined by the linear map $u \mapsto B_{u}$. Consider the matrices

$$
M_{i, j} \stackrel{\text { def }}{=}\left(\begin{array}{c|c|c}
1 & 0 & 0 \\
\hline 0 & 1_{n-d} & 0 \\
\hline 0 & 0 & 1_{d}+E_{i, j}
\end{array}\right),
$$


where $E_{i, j}$ is defined in the beginning of Sect. 4. These matrices represent elements of $\Sigma$ such that $M_{i, j}=M_{i, j}^{-1}$. A direct computation shows that

$$
M_{i, j}^{-1}\left(\begin{array}{c|c|c}
1 & u & w \\
\hline 0 & 1_{n-d} & B_{u} \\
\hline 0 & 0 & 1_{d}
\end{array}\right) M_{i, j}=\left(\begin{array}{c|c|c}
1 & u & w\left(1_{d}+E_{i, j}\right) \\
\hline 0 & 1_{n-d} & B_{u}\left(1_{d}+E_{i, j}\right) \\
\hline 0 & 0 & 1_{d}
\end{array}\right)
$$

so that if $\bar{T}$ is normal in $\Sigma$ then $B_{u}\left(1_{d}+E_{i, j}\right)=B_{u}$ for every $u \in U$ and $1 \leq i<j \leq d$. It follows that, for $1 \leq i \leq d-1$, the $i$-th column of $B_{u}$ is the zero column. The matrix

$$
N_{i, j} \stackrel{\text { def }}{=}\left(\begin{array}{c|c|c}
1 & 0 & 0 \\
\hline 0 & 1_{n-d}+E_{i, j} & 0 \\
\hline 0 & 0 & 1_{d}
\end{array}\right),
$$

where $1 \leq i<j \leq n-d$, represents an involution in $\Sigma$ and

$$
N_{i, j}^{-1}\left(\begin{array}{c|c|c}
1 & e_{h} & w \\
\hline 0 & 1_{n-d} & B_{e_{h}} \\
\hline 0 & 0 & 1_{d}
\end{array}\right) N_{i, j}=\left(\begin{array}{c|c|c}
1 & e_{h}\left(1_{n-d}+E_{i, j}\right) & w \\
\hline 0 & 1_{n-d} & \left(1_{n-d}+E_{i, j}\right) B_{e_{h}} \\
\hline 0 & 0 & 1_{d}
\end{array}\right) .
$$

If this matrix is in $\bar{T}$ and $h \neq i$, then $e_{h}\left(1_{n-d}+E_{i, j}\right)=e_{h}$, so that $\left(1_{n-d}+E_{i, j}\right) B_{e_{h}}=B_{e_{h}}$, which in turn implies that the $j$-th row of $B_{e_{h}}$ is the zero row for every $j \neq i$ and $j \neq h$. As seen in the proof of Theorem 5, the $h$-th row of $B_{e_{h}}$ is zero and the first row of $B_{e_{h}}$ equals the $h$-th row of $B_{e_{1}}$, which is zero. Therefore, $B_{e_{h}}=0$ for $3 \leq h \leq n$, so that $\left\langle e_{3}, \ldots, e_{n}\right\rangle \leq W$, which gives $d=\operatorname{dim}(W) \geq n-2$. Moreover, by Proposition 1 we have $d \leq n-2$, and therefore $d=n-2$. We can now apply Theorem 4 to obtain that $\bar{T} \unlhd \Sigma$ if and only if $T^{g}=\bar{T} \in\left\{T, T_{\Sigma}\right\}$.

With the same notation of the previous theorems we have the following corollary.

Corollory 3 Every $g \in N_{\text {Sym }(V)}(\Sigma) \backslash \operatorname{AGL}(V)$ interchanges by conjugation $T$ and $T_{\Sigma}$. Moreover, each element in $\operatorname{AGL}(V) \cap N_{\operatorname{Sym}(V)}\left(T_{\Sigma}\right)=\operatorname{AGL}(V) \cap \operatorname{AGL}(V)^{g}$ stabilises this action.

Proof Since the normaliser $N_{\mathrm{Sym}(V)}(\Sigma)$ permutes the normal elementary abelian regular subgroups of $\Sigma$ and AGL( $V)$ normalises $T$, the claim follows straightforwardly.

From Theorem 6 we can also conclude that second-maximal-intersection subgroups are characterised by the fact that their normalisers contain a Sylow 2-subgroup of AGL( $V)$.

Corollory 4 Let $g \in \operatorname{Sym}(V) \backslash \operatorname{AGL}(V)$ such that $T^{g}$ is an elementary abelian regular subgroup of $\operatorname{Sym}(V)$. If $|\operatorname{AGL}(V)|=2^{m} t$, with t an odd integer, then

$$
\left|T \cap T^{g}\right|=2^{n-2} \Longleftrightarrow 2^{m}|| \operatorname{AGL}(V) \cap \operatorname{AGL}(V)^{g} \mid .
$$

Proof If $\left|T \cap T^{g}\right|=2^{n-2}$, then by Theorem 3, both $T$ and $T^{g}$ are subgroups of AGL( $\left.V\right)$. Moreover, since $T$ is contained in every Sylow 2-subgroup of $\operatorname{AGL}(V)$, at least one of them, which we denote by $\Sigma$, contains both $T$ and $T^{g}$ as normal subgroups. Thus, $\Sigma \leq$ $\operatorname{AGL}(V) \cap \operatorname{AGL}(V)^{g}$. Conversely, if this is the case, $T$ and $T^{g}$, being contained in every Sylow 2-subgroup of their own normalisers, are distinct normal subgroups of $\Sigma$. Therefore, Theorems 4 and 6 yield $\left\{T, T^{g}\right\}=\left\{T, T_{\Sigma}\right\}$; hence, $\left|T \cap T^{g}\right|=2^{n-2}$.

It was already known to P. Hall (see, e.g. [10]) that if $\Xi$ is a Sylow 2-subgroup of $\operatorname{Sym}(V)$, then $N_{\text {Sym }(V)}(\Xi)=\Xi$. In the remainder of the paper, we establish a similar result for Sylow 2-subgroups of $\operatorname{AGL}(V)$. 
Theorem 7 If $\Sigma$ is a Sylow 2-subgroup of $\operatorname{AGL}(V)$, then

$$
\left[N_{\text {Sym }(V)}(\Sigma): \Sigma\right]=2 .
$$

Proof By Remark 4, there exists a flag $\{0\}=V_{0}<V_{1}<\ldots<V_{n}=V$ such that $\Sigma$ is the stabiliser by conjugation of $\sigma_{V_{0}}<\sigma_{V_{1}}<\ldots<\sigma_{V_{n}}=T$. By Theorem 6, for every $g \in N_{\operatorname{Sym}(V)}(\Sigma)$, we have $\sigma_{V_{i}}^{g^{2}}=\sigma_{V_{i}}$ for $1 \leq i \leq n$, and so $g^{2} \in \Sigma$. Hence, $N_{\operatorname{Sym}(V)}(\Sigma) / \Sigma$ has exponent equal to 2, i.e. it is an elementary abelian group. Let now $n, m \in N_{\operatorname{Sym}(V)}(\Sigma) \backslash \Sigma$. We have $T^{n m^{-1}}=T$, and so $n m^{-1} \in \Sigma$. Therefore, $\left|N_{\text {Sym }(V)}(\Sigma) / \Sigma\right| \leq 2$. On the other hand, since $\Sigma$ is properly contained in a Sylow 2-subgroup $\Xi$ of $\operatorname{Sym}(V)$, then it is well known that $\Sigma$ is properly contained in $N_{\Xi}(\Sigma) \leq N_{\operatorname{Sym}(V)}(\Sigma)$ and so $\left[N_{\operatorname{Sym}(V)}(\Sigma): \Sigma\right]=2$.

Remark 5 If $\operatorname{dim}(V)=n>2$, then $\operatorname{AGL}(V)$ is a subgroup of $\operatorname{Alt}(V)$, the alternating group on $V$. Indeed, we note that a subgroup $H$ of the symmetric group is contained in the alternating group if and only if one of its Sylow 2-subgroups is. The reason is that all the elements of odd order are contained in the alternating subgroup, and any other element is the product of a 2-element by an element of odd order. In our case, all the elements of $T$ are the product of an even number of transpositions, so that $T<\operatorname{Alt}(V)$. Hence, it suffices to show that any Sylow 2-subgroup of GL( $V)$ is contained in $\operatorname{Alt}(V)$. We recall that the matrices $1_{n}+E_{i, i+1}$, defined in the beginning of Sect. 4, generate a Sylow 2-subgroup of GL( $V)$. Each of such matrices fixes $2^{n-1}$ vectors, whereas it pairwise exchanges the remaining $2^{n}-2^{n-1}$ ones, i.e. as a permutation, it is the product of $2^{n-1}-2^{n-2}$ of transpositions, which is an even number.

Corollory 5 The normaliser in $\operatorname{Sym}(V)$ of each Sylow 2-subgroup $\Sigma$ of $\operatorname{AGL}(V)$ consists of even permutations. Thus, $N_{\mathrm{Sym}(V)}(\Sigma)=N_{\mathrm{Alt}(\mathrm{V})}(\Sigma)$.

Proof Let $\Sigma$ be a Sylow 2-subgroup of $\operatorname{AGL}(V)$. If $\Xi$ is a Sylow 2-subgroup of $\operatorname{Alt}(V)$ containing $\Sigma$, then $\Xi>\Sigma$, so that $N_{\Xi}(\Sigma)>\Sigma$. By Theorem 7, it follows that $N_{\text {Alt }(V)}(\Sigma) \geq$ $N_{\Xi}(\Sigma)=N_{\text {Sym }(V)}(\Sigma)$.

The result which follows is the counterpart in $\operatorname{AGL}(V)$ of the result due to P. Hall on the Sylow 2-subgroups of $\operatorname{Sym}(V)$. It also allows us to count the number of distinct Sylow 2-subgroups of $\operatorname{AGL}(V)$.

Theorem 8 If $\Sigma$ is a Sylow 2-subgroup of $\operatorname{AGL}(V)$, then $N_{\mathrm{AGL}(V)}(\Sigma)=\Sigma$. In particular,

$$
[\operatorname{AGL}(V): \Sigma]=\prod_{j=0}^{n-1}\left(2^{n-j}-1\right) .
$$

is the number of distinct Sylow 2-subgroups of $\mathrm{AGL}(V)$.

Proof By Theorem 7, if $|\operatorname{AGL}(V)|=2^{m} t$, with $t$ an odd integer, then we have $|\Sigma|=2^{m}$ and $\left|N_{\operatorname{Sym}(V)}(\Sigma)\right|=2^{m+1}$. Since $N_{\mathrm{AGL}(V)}(\Sigma) \leq \operatorname{AGL}(V)$ and $N_{\mathrm{AGL}(V)}(\Sigma) \leq N_{\operatorname{Sym}(V)}(\Sigma)$, then $\left|N_{\mathrm{AGL}(V)}(\Sigma)\right|=2^{m}$.

Finally, we use Theorem 6 to give an alternative proof of the following result.

Corollory 6 [[12]] The group AGL( $V)$ acts transitively by conjugation on the set of elementary abelian regular subgroups which intersect $T$ in a second-maximal subgroup of $T$. 
Proof It is enough to show that each elementary abelian regular group $\bar{T}$ such that $|T \cap \bar{T}|=$ $2^{n-2}$ is conjugated to $T_{S}$, where $S$ is the Sylow 2-subgroup of AGL( $\left.V\right)$ defined as in Eq. (3). By Proposition 3, there exists a Sylow 2-subgroup $\bar{S}$ of $\operatorname{AGL}(V)$ such that $\bar{T} \unlhd \bar{S}$. Moreover, $\bar{S}^{h}=S$ for some $h \in \operatorname{AGL}(V)$. By Theorem 6, it follows that $T_{S}=\bar{T}^{h} \unlhd \bar{S}^{h}$.

As a last consequence, the number of Sylow 2-subgroups of AGL( $V)$ which contain the same second-maximal-intersection subgroup as a normal subgroup can be determined.

Corollory 7 The number $s_{n}$ of Sylow 2-subgroups of AGL(V) which contain as a normal subgroup the same group $T^{g}$ such that $\operatorname{dim}(W)=n-2$, where $\sigma_{W}=T \cap T^{g}$ and $g \in$ $\operatorname{Sym}(V)$, is given by the formula:

$$
s_{n}=3 \prod_{j=3}^{n-1}\left(2^{n-j}-1\right) .
$$

Proof Let $|\operatorname{AGL}(V)|=2^{m} t$, with $t$ an odd integer. Firstly, we recall that $t$ is the integer displayed in Eq. (10). The claim follows from Corollary 2, since $s_{n}=t / t_{n}$, where $t_{n}$ is the number of elementary abelian regular subgroups in $\operatorname{AGL}(V)$ whose intersection with $T$ is a second-maximal subgroup of $T$.

\section{Conclusion and open problems}

We already mentioned that the conjugates in $\operatorname{Sym}(V)$ of $T$ are very important in the cryptanalysis of block ciphers. For this reason, a complete parameterisation of them in terms of the size of their intersection with $T$ is needed. Recall that the elements of such intersections are in one-to-one correspondence with the weak keys corresponding to the alternative operations. In this paper, the aforementioned problem has been addressed, both considering subgroups of $\operatorname{Sym}(V)$ and $\operatorname{AGL}(V)$, in the case where the weak-key subspace has dimension $n-1$ and $n-2$. This last case turns out to be one of the most relevant for cryptanalysis, for reasons whose description would lead us out of the scope of this work. We have computational evidence that also the case of lower dimensional weak-key spaces might be interesting from a cryptographic point of view, though it may require an entirely different technical approach.

Acknowledgements Funding was provided by Ministero dell' Istruzione, dell'Università e della Ricerca (IT) (Grant No. PRIN 2015TW9LSR).

\section{References}

1. Aragona, R., Calderini, M., Civino, R., Sala, M., Zappatore, I.: Wave-shaped round functions and primitive groups. Adv. Math. Commun. 13(1), 67-88 (2019)

2. Aragona, R., Caranti, A., Sala, M.: The group generated by the round functions of a GOST-like cipher. Annali di Matematica Pura ed Applicata (1923-) 196(1), 1-17 (2017)

3. Bosma, W., Cannon, J., Playoust, C.: The Magma algebra system. I. The user language. J. Symbolic Comput. 24(3-4), 235-265 (1997). Computational algebra and number theory (London, 1993)

4. Brunetta, C., Calderini, M., Sala, M.: On hidden sums compatible with a given block cipher diffusion layer. Discrete Math. 342(2), 373-386 (2019)

5. Bogdanov, A., Knudsen, L.R., Leander, G., Paar, C., Poschmann, A., Robshaw, M.J., Seurin, Y., Vikkelsoe, C.: PRESENT: An ultra-lightweight block cipher. In: International workshop on cryptographic hardware and embedded systems, pp. 450-466. Springer, (2007)

6. Biham, E., Shamir, A.: Differential cryptanalysis of DES-like cryptosystems. J. Crypt. 4(1), 3-72 (1991) 
7. Carlet, C.: Boolean functions for cryptography and error correcting codes. Boolean Models Methods Math. Comput. Sci. Eng. 2, 257-397 (2010)

8. Civino, R., Blondeau, C., Sala, M.: Differential attacks: using alternative operations. Designs Codes Cryptogr. 87(2-3), 225-247 (2019)

9. Caranti, A., Volta, F.D., Sala, M.: Abelian regular subgroups of the affine group and radical rings. Publ. Math. Debrecen 69(3), 297-308 (2006)

10. Carter, R., Fong, P.: The Sylow 2-subgroups of the finite classical groups. J. Algebra 1, 139-151 (1964)

11. Canteaut, A., Naya-Plasencia, M.: Structural weaknesses of permutations with a low differential uniformity and generalized crooked functions. In: Finite fields: Theory and applications-selected papers from the 9th international conference finite fields and applications. Contemp. Math. 518:55-71 (2010)

12. Calderini, M., Sala, M.: Elementary abelian regular subgroups as hidden sums for cryptographic trapdoors. ArXiv e-prints (2017)

13. Dixon, J.D.: Maximal abelian subgroups of the symmetric groups. Canad. J. Math. 23, 426-438 (1971)

14. Dolmatov, V.: Gost 28147-89: Encryption, decryption, and message authentication code (mac) algorithms. Tech. Rep. (2010)

15. Daemen, J., Rijmen, V.: The design of Rijndael: AES-the advanced encryption standard. Springer, Berlin (2013)

16. Liebeck, M.K., Praeger, C.E., Saxl, J.: A classification of the maximal subgroups of the finite alternating and symmetric groups. J. Algebra 111(2), 365-383 (1987)

17. Matsui, M.: Linear cryptanalysis method for DES cipher. In: Workshop on the theory and application of cryptographic techniques, Springer, pp. 386-397 (1993)

18. US Department of Commerce, National Bureau of Standards (1977) Data encryption standard. Federal information processing standards publication, vol 46, National Bureau of Standards, US Department of Commerce, Washington, p. 23

19. Nyberg, K.: Differentially uniform mappings for cryptography. In: Workshop on the theory and application of cryptographic techniques, Springer, pp. 55-64 (1993)

20. Seki H, Kaneko T.: Differential cryptanalysis of reduced rounds of GOST. In: International workshop on selected areas in cryptography, Springer, pp. 315-323 (2000)

Publisher's Note Springer Nature remains neutral with regard to jurisdictional claims in published maps and institutional affiliations. 